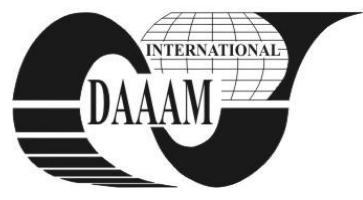

\title{
CONIC PACKAGES OSCILLATIONS
}

\section{PRACEK, S[tanislav]; SLUGA, F[ranci]; MOZINA, K[lemen] \& FRANKEN, G[regor]}

\begin{abstract}
Tension in the yarn and its oscillations during the over-end unwinding of the yarn from stationary packages depend on the unwinding speed, the shape and the winding type of the package, the air drag coefficient, and also the coefficient of friction between the yarn and the package. The yarn does not leave the surface package immediately at the unwinding point. Instead, it first slides on the surface and then lifts off to form the balloon.

Key words: conical packages, oscillations, winding angle, yarn unwinding, angular velocity
\end{abstract}

\section{INTRODUCTION}

When the yarn is being unwound from a cylindrical package, the angular velocity of the yarn forming the balloon depends on three parameters: the package radius, the unwinding velocity, and the winding angle. Particularly important is the last parameter, since it is closely related to the oscillations of the tension in the yarn (Kothari \& Leaf, 1979; Fraser et. al., 1992; Fraser, 1992; Clark et. al., 1998). In this paper we will investigate the unwinding from conic packages and the role of another parameter: the apex angle.

\section{THEORETICAL PART}

We study the unwinding from conic packages (Fig. 1). We denote the apex angle by $\alpha$ and the winding angle by $\phi$. In the present context, the winding angle is defined as the angle between the tangential line on the package surface which is perpendicular to the package axis and the line tangential to the yarn at the lift-off point. The smallest radius of the package is $\mathrm{c}_{0}$ and angle of conic $\alpha$.

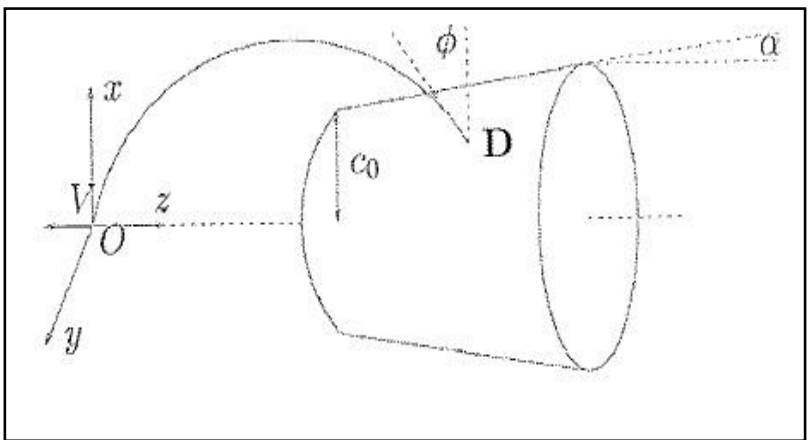

Fig. 1. Yarn unwinding from a conical package

In conical packages the relation between the angular velocity of the yarn during unwinding $\omega$, the unwinding speed $\mathrm{V}$ and the package radius $\mathrm{c}$ at the lift-off point, where the yarn lifts off from the package surface, is (Praček, 2002):

$$
\omega=\frac{V \mathrm{c} \text { o } \phi \mathrm{s}}{c(1-\mathrm{c} \text { ooss } \mathrm{i} \phi)}
$$

Here $\phi$ is the winding angle and $\alpha$ is the apex angle of the conical package
From the simplest theory of the yarn unwinding which doesn't take into account the air drag nor the Coriolis force, we know that the tension is proportional to the angular velocity squared

$$
T_{0} \propto \omega^{2}
$$

This expression is only approximately true. A better estimate could be obtained from the full equation of motion for the yarn, but no simple analytic expression can be obtained in this case. In addition, a balloon limiter is usually used during the unwinding. This device limits the radius of the balloon, thereby reducing the centrifugal force on the yarn in the balloon and consequently the tension is lower as well. For this reason we performed experimental measurements to determine the relation between the tension and the angular velocity. We measured tension for parallely wound cylindrical packages of different dimensions and for different unwinding velocities.

We performed a series of measurements for different unwinding velocities $V$ and package radii $c$.

In building this model we make a few assumptions:

1. The length of the yarn in the balloon has no effect on the tension.

2. We neglect the residual tension of yarn in the package, which is related to the stiffness of the winding.

3. The winding angle and the number of the threads are approximately constant in the number of the layers whose unwinding will be simulated.

\section{PRACTICAL PART}

We now present the results of the simulations. In Fig. 2 we show the tension during unwinding from conic packages of different apex angles. As expected on basis of Eq. (1), in the range of apex angles of conic packages, the effect of package shape is rather small.

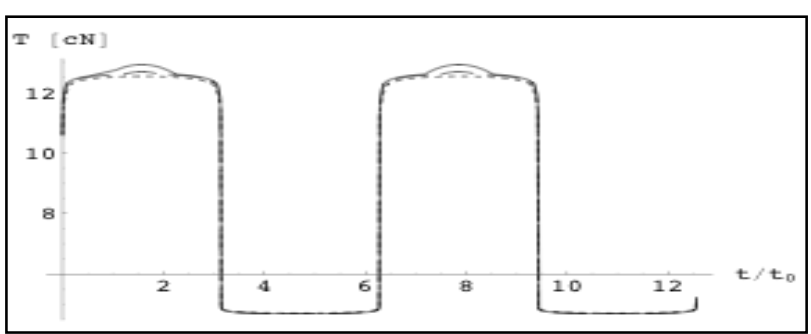

Fig. 2: Tension for conic packages of radius $c=200 \mathrm{~mm}$ and winding angle $\phi=10$ at unwinding velocity $V=2000 \mathrm{~m} / \mathrm{min}$ for different apex angles. $\alpha=0^{\circ}$ solid line, $\alpha=5^{\circ}$ dashed line, $\alpha=10^{\circ}$ dotted line

The oscillations of the tension are related to the oscillations in the angular velocity of the yarn. The amplitude of the angular velocity oscillations can be obtained as 


$$
\begin{aligned}
& \Delta \omega=\omega_{\max }-\omega_{\min }=\frac{V}{c} \frac{\cos \phi}{1-\sin \phi}-\frac{V}{c} \frac{\cos (-\phi)}{1-\sin (-\phi)} \\
& =\frac{2 V}{c} \tan \phi .
\end{aligned}
$$

In the region of interest, i.e. for $\phi<25^{\circ}$, we can approximate $\tan \phi \sim \phi$, so we have

$$
\Delta \omega \approx \frac{2 V \phi}{c} .
$$

According to this expression, the amplitude of angular velocity oscillations is approximately proportional to the unwinding velocity and the winding angle and inversely proportional to the package radius.

In Fig. 3 we plot the amplitude of oscillations of the tension as a function of the winding angle and the package radius for a constant unwinding velocity $V=2000 \mathrm{~m} / \mathrm{min}$. Oscillations become very large for increasing value of the winding angle. For packages of small radius, $c=70 \mathrm{~mm}$, unwinding is only safe for winding angle below $\phi=5^{\circ}$.

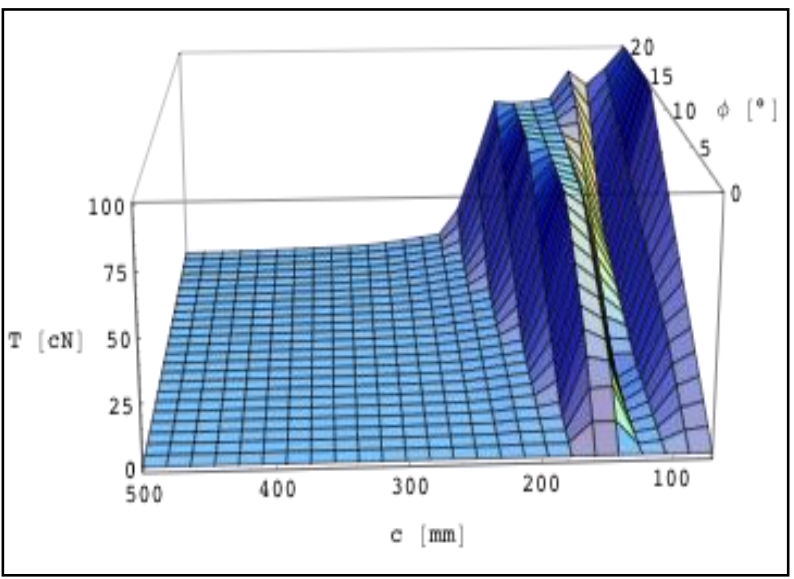

Fig. 3. Amplitude of oscillations as a function of the winding angle $\phi$ and the package radius $\mathrm{c}$ for constant unwinding velocity $V=2000 \mathrm{~m} / \mathrm{min}$

In Fig. 4 we show the amplitude of oscillations as a function of package radius and the unwinding velocity for a constant winding angle $\phi=5^{\circ}$. The curves of constant amplitude are simply straight lines, as expected, since at constant winding angle the amplitude depends only on the ratio $V / c$. This is a useful rule of thumb. We can opt for small package radii and reduce the unwinding speed, however for unwinding at large unwinding speeds it is necessary to increase the radius.

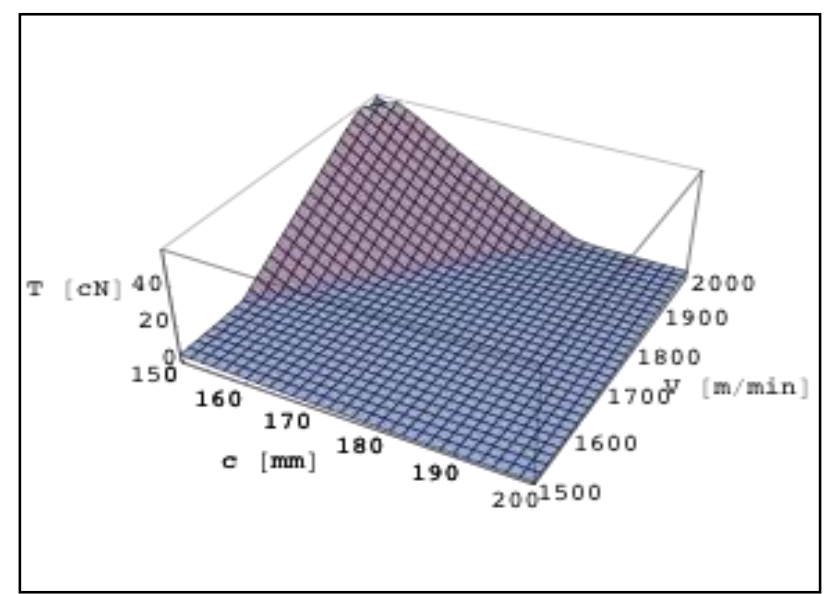

Fig. 4. Amplitude of oscillations as a function of package radius $c$ and the unwinding velocity $V$ for the winding angle of $\phi=5^{\circ}$

\section{CONCLUSION}

We have shown that a combination of theoretical modelling and empirically determined relation between the yarn tension and the angular velocity of the yarn.

We have reached the following conclusions:

- Oscillations of tension are smaller in packages with small winding angle (parallel-wound packages), but for such packages yarn slips can occur during unwinding.

- Alternatively, the oscillations of tension can be reduced if only those layers of yarn that are unwound backwards are parallely wound.

\section{REFERENCES}

Kothari,V.K.,Leaf G.A.V. (1979). The unwinding of yarns from packages, PartII: The theory of yarnunwinding.J.Text.Inst 70 (3)95-105

Fraser, W. B., Ghosh, T. K., Batra, S. K. (1992). On unwinding yarn from cylindrical package. Proc. R. Soc. Lond. A, 436 479-498

Fraser, W. B., (1992). The effect of yarn elasticity on an unwinding ballon. J. Tex. Inst, 83 603-613

J.D.Clark, W.B.Fraser, R. Sharma and C.D.Rahn. (1998). The dynamic response of a ballooning yarn:theory and experiment. Proc. R. Soc. Lond. A, 454 2767-2789

Praček, S. (2002). Modification of yarn unwinding dynamics. Dissertation, University of Ljubljana 